\title{
Contact models for very loose granular materials
}

\author{
Stefan Luding \\ Particle Technology, Nanostructured Materials, DelftChemTech, \\ TU Delft, Julianalaan 136, 2628 BL Delft, The Netherlands \\ e-mail: s.luding@tudelft.nl
}

June 28, 2006

\begin{abstract}
One challenge of todays research on particle systems is the realistic simulation of granular materials consisting of many thousands of particles with peculiar contact interactions. In this study, molecular dynamics (MD, also called discrete element method, DEM) is introduced for the simulation of many-particle systems. A wide class of realistic contact models is presented, involving dissipation, adhesion, plastic deformation, friction, rolling- and torsion resistance.

The effect of the contact properties on a simple compaction test is discussed with the goal to achieve as small as possible packing densities. With contact forces only, packing volume fractions down to 0.42 can be achieved, while somewhat longer ranged adhesion forces allow for volume fractions as low as 0.34 .

Keywords

granular materials, molecular dynamics (MD), discrete element model (DEM), compaction, friction, rolling- and torsion resistance, adhesion, plastic deformation, low density compaction
\end{abstract}

\section{Introduction}

Molecular Dynamics (MD) or Discrete Element Models (DEM) are solving the equations of motion for all particles in a system, where the contact forces are the only physical laws that have to be defined beforehand. A straightforward approach towards the understanding of the macroscopic material behavior of fine granular materials like powders, by just modeling and simulating all particles in a big system, is not possible due to the huge number of particles typically involved. Therefore, one has to reduce the size of the system under consideration, so that a microscopic simulation of all particles becomes feasible. The goal is to understand the macroscopic flow behavior from such small scale models - both from simulations and from experiment - and to provide (macroscopic) constitutive relations for standard tools like the finite-element method (FEM) suited to deal with large-scale systems. Tools to perform a so-called micro-macro transition are developed [1], and the goal is to relate the macroscopic flow behavior to the microscopic contact properties.

For powders, as an example, the particle properties and interaction laws are inserted into a discrete particle molecular dynamics and lead to the collective behavior 
of the dissipative, frictional, adhesive many-particle system. From the particle simulation, one can extract, e.g., the coordination number or the pressure of the system as a function of density. In the following, normal and tangential interactions, like adhesion, plastic contact deformation, friction, rolling- and torsion resistance are discussed for spherical model particles. Examples of a compression test are presented for which the previously defined contact model parameters are varied.

\section{The Soft Particle Molecular Dynamics Method}

Particle simulations like MD or DEM [1-7] can complement experiments on small "representative volume elements" (REV). Alternative methods like contact dynamics (CD) or cell- and lattice gas-methods are not discussed here.

\subsection{Discrete Particle Model}

The elementary units of granular materials are mesoscopic grains, which deform under stress. Since the realistic modeling of the deformations of the particles is much too complicated, we relate the interaction force to the overlap $\delta$ of two particles, see Fig. 1. In tangential direction, some forces also depend on the tangential displacement since the beginning of the contact, like it is the case for torques, e.g., due to friction or rolling resistance. Note that the evaluation of the inter-particle forces based on the overlap may not be sufficient to account for the inhomogeneous stress distribution inside the particles and possible multi-contact effects. Consequently, the results presented here are of the same quality as the simplifying assumptions about the force-overlap relations made.
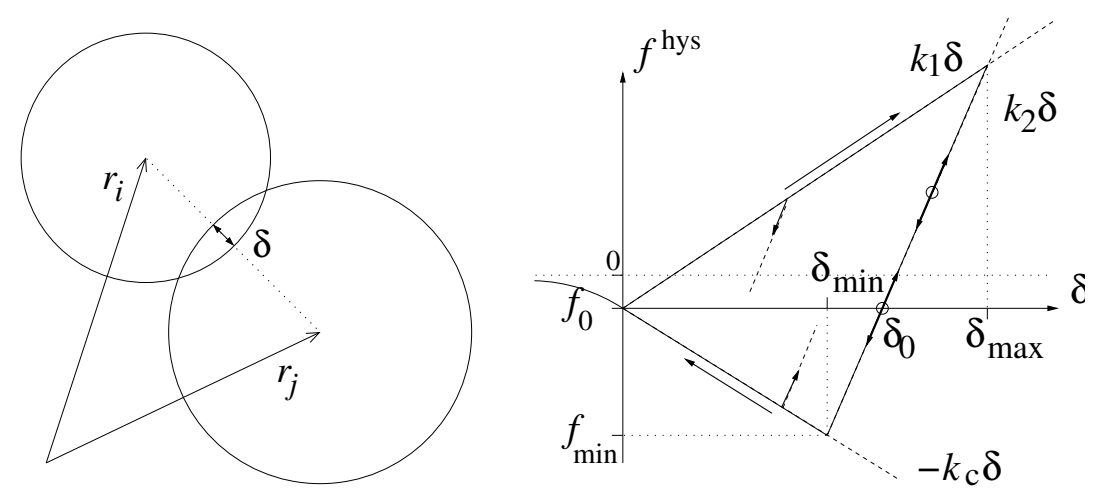

Figure 1: (Left) Two particle contact with overlap $\delta$ in normal direction. (Right) Schematic graph of the piece-wise linear, hysteretic, adhesive force-displacement model in normal direction.

\subsection{Equations of Motion}

If all forces $\boldsymbol{f}_{i}$ acting on the particle $i$, either from other particles, from boundaries or from external forces, are known, the problem is reduced to the integration of Newton's 
equations of motion for the translational and rotational degrees of freedom:

$$
m_{i} \frac{\mathrm{d}^{2}}{\mathrm{~d} t^{2}} \boldsymbol{r}_{i}=\boldsymbol{f}_{i}+m_{i} \boldsymbol{g}, \quad \text { and } \quad I_{i} \frac{\mathrm{d}^{2}}{\mathrm{~d} t^{2}} \boldsymbol{\varphi}_{i}=\boldsymbol{q}_{i}
$$

with the mass $m_{i}$ of particle $i$, its position $\boldsymbol{r}_{i}$ the total force $\boldsymbol{f}_{i}=\sum_{c} \boldsymbol{f}_{i}^{c}$ acting on it due to contacts with other particles or with the walls, the acceleration due to volume forces like gravity $\boldsymbol{g}$, the spherical particles moment of inertia $I_{i}$, its angular velocity $\boldsymbol{\omega}_{i}=\mathrm{d} \boldsymbol{\varphi}_{i} / \mathrm{d} t$ and the total torque $\boldsymbol{q}_{i}$.

The equations of motion are thus a system of $\mathcal{D}+\mathcal{D}(\mathcal{D}-1) / 2$ coupled ordinary differential equations to be solved in $\mathcal{D}$ dimensions, with $\mathcal{D}=2$ or 3 . With tools from numerical integration, as nicely described in textbooks as $[8,9]$, this is a straightforward exercise. The typically short-ranged interactions in granular media, allow for further optimization by using linked-cell or alternative methods $[8,9]$ in order to make the neighborhood search more efficient. In the case of long-range interactions, (e.g., charged particles or van der Waals type forces) this is not possible anymore, so that either a cut-off or more advanced methods for optimization have to be applied - for the sake of brevity, we restrict ourselves to the cut-off method below.

\subsection{Normal Contact Force Laws}

Two spherical particles $i$ and $j$, with radii $a_{i}$ and $a_{j}$, respectively, interact only if they are in contact so that their overlap

$$
\delta=\left(a_{i}+a_{j}\right)-\left(\boldsymbol{r}_{i}-\boldsymbol{r}_{j}\right) \cdot \boldsymbol{n}
$$

is positive, $\delta>0$, with the unit vector $\boldsymbol{n}=\boldsymbol{n}_{i j}=\left(\boldsymbol{r}_{i}-\boldsymbol{r}_{j}\right) /\left|\boldsymbol{r}_{i}-\boldsymbol{r}_{j}\right|$ pointing from $j$ to $i$. The force on particle $i$, from particle $j$, at contact $c$, can be decomposed into a normal and a tangential part as $\boldsymbol{f}^{c}:=\boldsymbol{f}_{i}^{c}=f^{n} \boldsymbol{n}+f^{t} \boldsymbol{t}$, where $\boldsymbol{n} \cdot \boldsymbol{t}=0$.

\subsubsection{Linear Normal Contact Model}

The simplest normal contact force model, which takes into account excluded volume and dissipation, involves a linear repulsive and a linear dissipative force

$$
f^{n}=k \delta+\gamma_{0} v_{n}
$$

with a spring stiffness $k$, a viscous damping $\gamma_{0}$, and the relative velocity in normal direction $v_{n}=-\boldsymbol{v}_{i j} \cdot \boldsymbol{n}=-\left(\boldsymbol{v}_{i}-\boldsymbol{v}_{j}\right) \cdot \boldsymbol{n}=\dot{\delta}$.

This so-called linear spring dashpot (LSD) model allows to view the particle contact as a damped harmonic oscillator, for which the half-period of a vibration around an equilibrium position with a certain contact force, can be computed [10]. The typical response time on the contact level is

$$
t_{c}=\frac{\pi}{\omega}, \quad \text { with } \omega=\sqrt{\left(k / m_{12}\right)-\eta_{0}^{2}},
$$

the eigenfrequency of the contact, the rescaled damping coefficient $\eta_{0}=\gamma_{0} /\left(2 m_{i j}\right)$, and the reduced mass $m_{i j}=m_{i} m_{j} /\left(m_{i}+m_{j}\right)$. From the solution of the equation of a half period of the oscillation, one also obtains the coefficient of restitution

$$
r=v_{n}^{\prime} / v_{n}=\exp \left(-\pi \eta_{0} / \omega\right)=\exp \left(-\eta_{0} t_{c}\right),
$$


which quantifies the ratio of normal relative velocities after (primed) and before (unprimed) the collision. For a more detailed discussion of this and other, more realistic, non-linear contact models, see Ref. [10].

The contact duration in Eq. (4) is also of practical technical importance, since the integration of the equations of motion is stable only if the integration time-step $\Delta t_{\mathrm{MD}}$ is much smaller than $t_{c}$. Note that $t_{c}$ depends on the magnitude of dissipation: In the extreme case of an overdamped spring, $t_{c}$ can become very large (which would render the contact behavior artificial [19]). Therefore, the use of neither too weak nor too strong dissipation is recommended.

\subsubsection{Adhesive, Plastic, Hysteretic Normal Contact Model}

Here we apply a variant of the linear hysteretic spring model [10-12], as an alternative to the frequently applied spring-dashpot models. This model is the simplest version of some more complicated nonlinear-hysteretic force laws [11,13,14], which reflect the fact that at the contact point, plastic deformations may take place and attractive (adhesive) forces exist. The adhesive, plastic (hysteretic) force-law can be written as

$$
f^{\text {hys }}=\left\{\begin{array}{lll}
k_{1} \delta & \text { for loading, } & \text { if } k_{2}\left(\delta-\delta_{0}\right) \geq k_{1} \delta \\
k_{2}\left(\delta-\delta_{0}\right) & \text { for un/reloading, } & \text { if } k_{1} \delta>k_{2}\left(\delta-\delta_{0}\right)>-k_{c} \delta \\
-k_{c} \delta & \text { for unloading, } & \text { if }-k_{c} \delta \geq k_{2}\left(\delta-\delta_{0}\right)
\end{array}\right.
$$

with $k_{1} \leq k_{2}$, see Fig. 1 . During the initial loading the force increases linearly with the overlap $\delta$, until the maximum overlap $\delta_{\max }$ is reached (which has to be kept in memory as a history parameter). The line with slope $k_{1}$ thus defines the maximum force possible for a given $\delta$. During unloading the force drops from its value at $\delta_{\max }$ down to zero at overlap $\delta_{0}=\left(1-k_{1} / k_{2}\right) \delta_{\max }$, on the line with slope $k_{2}$, so that $\delta_{0}$ resembles the plastic contact deformation. Reloading at any instant leads to an increase of the force along the line with slope $k_{2}$, until the maximum force is reached; for still increasing $\delta$, the force follows again the line with slope $k_{1}$ and $\delta_{\max }$ has to be adjusted accordingly.

Unloading below $\delta_{0}$ leads to negative, attractive forces until the minimum force $-k_{c} \delta_{\min }$ is reached at the overlap $\delta_{\min }=\left(k_{2}-k_{1}\right) \delta_{\max } /\left(k_{2}+k_{c}\right)$. This minimum force, i.e., the maximum attractive force, is obtained as a function of the model parameters $k_{1}, k_{2}, k_{c}$, and the history parameter $\delta_{\max }$. Further unloading leads to attractive forces $f^{\text {hys }}=-k_{c} \delta$ on the adhesive branch with slope $-k_{c}$. The highest possible attractive force, for given $k_{1}$ and $k_{2}$, is reached for $k_{c} \rightarrow \infty$, so that $f_{\max }^{\text {hys }}=-\left(k_{2}-k_{1}\right) \delta_{\max }$. Since this would lead to a discontinuity at $\delta=0$, it is avoided by using finite $k_{c}$.

The lines with slope $k_{1}$ and $-k_{c}$ define the range of possible force values and departure from these lines takes place in the case of loading and unloading, respectively. Between these two extremes, unloading and reloading follow the line with slope $k_{2}$. Possible equilibrium states are indicated as circles in Fig. 1, where the upper and lower circle correspond to a pre-stressed and stress-free state, respectively. Small perturbations lead, in general, to small deviations along the line with slope $k_{2}$ as indicated by the arrows in Fig. 1.

A non-linear un/reloading behavior would be more realistic, however, due to a lack of detailed experimental informations, the piece-wise linear model is used as a compromise. One reasonable refinement, which accounts for an increasing stiffness with deformation, is a $k_{2}$ value dependent on the maximum overlap. This also implies rela- 
tively small and large plastic deformations for weak and strong contact forces, respectively. The model, as proposed recently [15], requires an additional model parameter,

$$
\delta_{\max }^{*}=\frac{k_{2}}{k_{2}-k_{1}} \phi_{f} \frac{a_{1}+a_{2}}{2},
$$

with the dimensionless plasticity depth, $\phi_{f}$, defined relative to the average radius. If the penetration is larger than a fraction $\phi_{f}$ of the (average) particle radius, the constant stiffness $k_{2}$ is used ${ }^{1}$. For smaller penetration, $k_{2}\left(\delta_{\max }\right)$ interpolates between $k_{1}$ to $k_{2}$ :

$$
k_{2}\left(\delta_{\max }\right)=\left\{\begin{array}{ll}
k_{2} & \text { if } \delta_{\max } \geq \delta_{\max }^{*} \\
k_{1}+\left(k_{2}-k_{1}\right) \delta_{\max } / \delta_{\max }^{*} & \text { if } \delta_{\max }<\delta_{\max }^{*}
\end{array},\right.
$$

and $k_{2}$ in Eq. (6) is replaced by $k_{2}\left(\delta_{\max }\right)$ from Eq. (8).

While in the case of collisions of particles with large relative velocities and thus deformations, dissipation takes place due to the hysteretic nature of the force-law, reasonably strong dissipation of small amplitude deformations is achieved by adding the viscous, velocity dependent dissipative force from Eq. (3) to the hysteretic force, such that $f^{n}=f^{\text {hys }}+\gamma_{0} v_{n}$.

In summary, the adhesive, plastic, hysteretic normal contact model contains the five parameters $k_{1}, k_{2}, k_{c}, \phi_{f}$, and $\gamma_{0}$ that respectively account for loading-reloadingstiffness and plastic deformation, adhesion, plastic overlap-range of the model, and viscous dissipation .

\subsubsection{Long Range Normal Forces}

Medium range van der Waals forces can be taken into account in addition to the hysteretic force such that $f^{n}=f^{\text {hys }}+\gamma_{0} v_{n}+f^{\mathrm{vdW}}$ with, for example, a Lennard-Jones Potential, leading to the force as function of distance:

$$
f^{\mathrm{vdW}}(r)=-\left(4 \varepsilon / r_{0}\right)\left[6\left(r_{0} / r\right)^{7}-12\left(r_{0} / r\right)^{13}\right] .
$$

In order to have a continuous force-displacement relation and to limit the range of the force, usually, a cut-off is introduced, so that

$$
f^{\mathrm{vdW}}=f^{\mathrm{vdW}}(r)-f^{\mathrm{vdW}}\left(r_{c}\right), \text { for } r<r_{c},
$$

and $f^{\mathrm{vdW}}=0$ elsewhere. The new parameters necessary for this force are an energy scale $\varepsilon$, a typical length scale $r_{0}$ and the cut-off length $r_{c}$. As long as $r_{c}$ is not too large as compared to the particle diameter, the methods for short range interactions still can be applied to such a medium range interaction model - only the linked cells have to be larger than twice the cut-off radius. When $r_{0}$ is smaller than the particle diameter, the repulsive part of the force becomes irrelevant due to the repulsive contact model.

\subsection{Tangential Contact Force Laws}

For the tangential degrees of freedom, there are three different force- and torque-laws to be implemented: (i) friction, (ii) rolling resistance, and (iii) torsion resistance. For friction, the relative tangential velocity of the contact points,

$$
\boldsymbol{v}_{t}=\boldsymbol{v}_{i j}-\boldsymbol{n}\left(\boldsymbol{n} \cdot \boldsymbol{v}_{i j}\right),
$$

\footnotetext{
${ }^{1}$ Note that a limit to the slope $k_{2}$ is needed for practical reasons. If $k_{2}$ would not be limited, the contact duration could become very small so that the time step would have to be reduced below reasonable values.
} 
is to be considered for the force and torque computations in the following subsections, with the total relative velocity of the particle surfaces at the contact

$$
\boldsymbol{v}_{i j}=\boldsymbol{v}_{i}-\boldsymbol{v}_{j}+a_{i} \boldsymbol{n} \times \boldsymbol{\omega}_{i}+a_{j} \boldsymbol{n} \times \boldsymbol{\omega}_{j} .
$$

Thus, the frictional force and torque are active when the two particles are rotating in parallel. The forces on the contacting particles are equally strong, but opposite, i.e., $\boldsymbol{f}_{j}^{t}=-\boldsymbol{f}_{i}^{t}$, while the corresponding torques are parallel but not necessarily equal in magnitude, i.e., $\boldsymbol{q}_{i}^{\text {friction }}=-a_{i} \boldsymbol{n} \times \boldsymbol{f}_{i}$, and $\boldsymbol{q}_{j}^{\text {friction }}=\left(a_{j} / a_{i}\right) \boldsymbol{q}_{i}^{\text {friction }}$. Note that forces and torques together conserve the total angular momentum, see Ref. [10].

For rolling resistance, the rolling velocity

$$
\boldsymbol{v}_{r}=-a_{i} \boldsymbol{n} \times \boldsymbol{\omega}_{i}+a_{j} \boldsymbol{n} \times \boldsymbol{\omega}_{j},
$$

is to be considered, which activates torques when two particles are rotating anti-parallel with spins in the tangential plane. These torques act against rolling and are equal in magnitude and opposite in direction, i.e., $\boldsymbol{q}_{i}^{\text {rolling }}=-\boldsymbol{q}_{j}^{\text {rolling }}=a_{i j} \boldsymbol{n} \times \boldsymbol{f}_{r}$, with the reduced radius $a_{i j}=2 a_{i} a_{j} /\left(a_{i}+a_{j}\right)$, and the quasi-force $\boldsymbol{f}_{r}$. This quasi-force is equal for both particles and does not act on the center of mass so that the total momentum (translational and angular) is conserved.

For torsion resistance, the relative spin along the normal direction

$$
\boldsymbol{v}_{o}=\left(a_{i} \boldsymbol{n} \cdot \boldsymbol{\omega}_{i}-a_{j} \boldsymbol{n} \cdot \boldsymbol{\omega}_{j}\right) \boldsymbol{n},
$$

is to be considered, which activates torques when two particles are rotating anti-parallel with spins parallel to the normal direction. These torsion torques are also equal in magnitude and directed opposite in direction, i.e., $\boldsymbol{q}_{i}^{\text {torsion }}=-\boldsymbol{q}_{j}^{\text {torsion }}=a_{i j} \boldsymbol{f}_{o}$, with the quasi-force $f_{o}$ that also does not change the translational momentum, but results in torques that conserves the total angular momentum.

The implementation of the tangential force computations for $\boldsymbol{f}_{t}, \boldsymbol{f}_{r}$, and $\boldsymbol{f}_{o}$ as based on $\boldsymbol{v}_{t}, \boldsymbol{v}_{r}$, and $\boldsymbol{v}_{o}$, respectively, is assumed to be identical, i.e., even the same subroutine is used, however, with different parameters as specified below. The difference is that friction leads to a force in the tangential plane (changing both translational and angular momentum), while rolling- and torsion-resistance lead to a quasi-forces in the tangential plane and the normal direction, respectively, changing angular momentum only. For more details on tangential contact models, friction, rolling and torsion, see Refs. [17, 18].

\subsubsection{Frictional Tangential Contact Model}

The tangential force is coupled to the normal force via Coulombs law, i.e. $f^{t} \leq \mu^{s} f^{n}$, where for the limit case one has dynamic friction with $f^{t}=\mu^{d} f^{n}$. The dynamic and the static friction coefficients follow, in general, the relation $\mu^{d} \leq \mu^{s}$. The static situation requires an elastic spring in order to allow for a restoring force, i.e. a non-zero remaining tangential force in static equilibrium due to activated Coulomb friction.

If a repulsive contact is established, and thus one has $f^{n}>0$, the tangential force is active. In the presence of adhesion, Coulombs law has to be slightly modified in so far that $f^{n}$ is replaced by $f^{n}+k_{c} \delta$. With other words, the reference criterion for a contact is no longer the zero force level, but it is the adhesive, attractive force level along $-k_{c} \delta$. Coulombs law in the presence of adhesion thus reads $f^{t} \leq \mu^{s}\left(f^{n}+k_{c} \delta\right)$ for the static case and $f^{t}=\mu^{d}\left(f^{n}+k_{c} \delta\right)$ for the dynamic, sliding case. 
If a contact is active, one has to project (or better rotate) the tangential spring into the actual tangential plane, since the frame of reference of the contact may have rotated since the last time-step. The new tangential spring is:

$$
\boldsymbol{\xi}=\boldsymbol{\xi}^{\prime}-\boldsymbol{n}\left(\boldsymbol{n} \cdot \boldsymbol{\xi}^{\prime}\right),
$$

where $\xi^{\prime}$ is the old spring from the last iteration. This action is relevant only for an already existing spring; if the spring is new, the tangential spring-length is zero anyway, however, its change is well defined even for the first, initiation step. In order to compute the changes of the tangential spring, a tangential test-force is first computed as the sum of the tangential spring force and a tangential viscous force (in analogy to the normal viscous force)

$$
\boldsymbol{f}_{0}^{t}=-k_{t} \boldsymbol{\xi}-\gamma_{t} \boldsymbol{v}_{t},
$$

with the tangential spring stiffness $k_{t}$, the tangential dissipation parameter $\gamma_{t}$, and $\boldsymbol{v}_{t}$ from Eq. (11). As long as $\left|\boldsymbol{f}_{0}^{t}\right| \leq f_{C}^{s}$, with $f_{C}^{s}=\mu^{s}\left(f^{n}+k_{c} \delta\right)$, one has static friction and, on the other hand, if the limit $\left|\boldsymbol{f}_{0}^{t}\right|>f_{C}^{s}$ is reached, sliding friction is active with magnitude $f_{C}^{d}=\mu^{d}\left(f^{n}+k_{c} \delta\right.$ ). (As soon as $\left|\boldsymbol{f}_{0}^{t}\right|$ becomes smaller than $f_{C}^{d}$, static friction is active again.) In the former, static case, the tangential spring is incremented

$$
\boldsymbol{\xi}^{\prime}=\boldsymbol{\xi}+\boldsymbol{v}_{t} \Delta t_{\mathrm{MD}},
$$

to be used in the next iteration in Eq. (15), and the force $\boldsymbol{f}^{t}=\boldsymbol{f}_{0}^{t}$ from Eq. (16) is used. In the latter, sliding case, the tangential spring is adjusted to a length which is consistent with Coulombs condition

$$
\boldsymbol{\xi}^{\prime}=-\frac{1}{k_{t}} f_{C}^{d} \boldsymbol{t},
$$

with the tangential unit vector, $\boldsymbol{t}=\boldsymbol{f}_{0}^{t} /\left|\boldsymbol{f}_{0}^{t}\right|$, defined by Eq. (16), and thus the magnitude of the Coulomb force is used. Inserting $\boldsymbol{\xi}^{\prime}$ from Eq. (18) into Eq. (16) leads to $\boldsymbol{f}_{0}^{t} \approx f_{C}^{d} \boldsymbol{t}-\gamma_{t} \boldsymbol{v}_{t}$. Note that $\boldsymbol{f}_{0}^{t}$ and $\boldsymbol{v}_{t}$ are not necessarily parallel in three dimensions. However, the mapping in Eq. (18) works always, rotating the new spring such that the direction of the frictional force is unchanged and, at the same time, limiting the spring in length according to Coulombs law. In short notation the tangential contact law reads

$$
\boldsymbol{f}^{t}=f^{t} \boldsymbol{t}=+\min \left(f_{C},\left|\boldsymbol{f}_{0}^{t}\right|\right) \boldsymbol{t},
$$

where $f_{C}$ follows the static/dynamic selection rules described above. The torque on a particle due to frictional forces at this contact is $q^{\text {friction }}=\boldsymbol{l}_{i}^{c} \times \boldsymbol{f}_{i}^{c}$, where $\boldsymbol{l}_{i}^{c}$ is the branch vector, connecting the center of the particle with the contact point.

The four parameters for the friction law are $k_{t}, \mu_{s}, \phi_{d}=\mu_{d} / \mu_{s}$, and $\gamma_{t}$, accounting for tangential stiffness, the static friction coefficient, the dynamic friction ratio, and tangential viscosity, respectively. Note that the tangential force described above is identical to the classical Cundall-Strack spring only in the limits $\mu=\mu^{s}=\mu^{d}$, i.e., $\phi_{d}=1$, and $\gamma_{t}=0$. The sequence of computations and the definitions and mappings into the tangential direction can be used in three dimensions as well as in two.

\subsubsection{Rolling Resistance Contact Model}

The three new parameters for rolling resistance are $k_{r}, \mu_{r}$, and $\gamma_{r}$, while $\phi_{d}$ is used from the friction law. The new parameters account for rolling stiffness, the static rolling "friction" coefficient, and rolling viscosity, respectively. In the subroutine called, the rolling velocity $\boldsymbol{v}_{r}$ is used instead of $\boldsymbol{v}_{t}$ and the computed quasi-force $\boldsymbol{f}_{r}$ is used to compute the torques, $q^{\text {rolling }}$, on the particles, see above. 


\subsubsection{Torsion Resistance Contact Model}

The three new parameters for rolling resistance are $k_{o}, \mu_{o}$, and $\gamma_{o}$, while $\phi_{d}$ is used from the friction law. The new parameters account for torsion stiffness, the static torsion "friction" coefficient, and torsion viscosity, respectively. In the subroutine, the torsion velocity $\boldsymbol{v}_{o}$ is used instead of $\boldsymbol{v}_{t}$ and the projection is a projection along the normal unit-vector. The computed quasi-force $f_{o}$ is then used to compute the torques, $q^{\text {torsion }}$, on the particles.

\subsection{Background Friction}

Note that the viscous dissipation takes place in a two-particle contact. In the bulk material, where many particles are in contact with each other, this dissipation mode is very inefficient for long-wavelength cooperative modes of motion $[16,19]$. Therefore, an additional damping with the background can be introduced, so that the total force on particle $i$ is

$$
\boldsymbol{f}_{i}=\sum_{j}\left(f^{n} \boldsymbol{n}+f^{t} \boldsymbol{t}\right)-\gamma_{b} \boldsymbol{v}_{i}
$$

and the total torque

$$
\boldsymbol{q}_{i}=\sum_{j}\left(\boldsymbol{q}^{\text {friction }}+\boldsymbol{q}^{\text {rolling }}+\boldsymbol{q}^{\text {torsion }}\right)-\gamma_{b r} \boldsymbol{\omega}_{i},
$$

with the damping artificially enhanced in the spirit of a rapid relaxation and equilibration. The sum in Eqs. (20) and (21) takes into account all contact partners $j$ of particle $i$, but the background dissipation can be attributed to the medium between the particles. Note that the effect of $\gamma_{b}$ and $\gamma_{b r}$ should be checked for each simulation in order to exclude artificial over-damping.

\section{Compaction Simulation Results}

In this section, a compression test is presented, where the particles are positioned on a square-lattice in a cubic system with periodic boundary conditions, in order to avoid wall effects. The system is first allowed to evolve to a disordered state, by attributing random velocities to all particles. The density is then increased by slowly increasing the particle size while the system volume $V=L^{3}$, with $L=0.025 \mathrm{~m}$, is kept constant, and reporting density and energies.

\subsection{Model Parameters}

The systems examined in the following contain $N=1728$ particles with equal radii $a$. In the simulation, the radii change according to the relation

$$
\frac{d a}{d t}=g_{a},
$$

with the growth rate $g_{a}=2 \cdot 10^{-7} \mathrm{~ms}^{-1}$, if not explicitly specified. The growth is stopped when a target volume fraction $\nu=N V(a) / V$ is reached, with the particle volume $V(a)=(4 / 3) \pi a^{3}$. The particle mass $m(a)=\rho V(a)$, with the material density $\rho$, changes with the radius during the growth period. 


\begin{tabular}{|ll||l|l|}
\hline Property & Symbol & Values & $t$-rescaled \\
\hline \hline Time Unit & $t_{u}$ & $1 \mathrm{~s}$ & $1 \mu \mathrm{s}$ \\
\hline Initial particle radius & $a_{0}$ & $0.5 \mu \mathrm{m}$ & \\
Growth rate & $g_{a}$ & $0.2 \mu \mathrm{m} / \mathrm{s}$ & $0.2 \mathrm{~m} / \mathrm{s}$ \\
Particle radius & $a(t)=a_{0}+g_{a} t$ & & \\
Material density & $\rho$ & $2000 \mathrm{~kg} / \mathrm{m}^{3}$ & \\
\hline Elastic stiffness & $k=k_{2}$ & $10^{-7} \mathrm{~kg} / \mathrm{s}^{2}$ & $10^{5} \mathrm{~kg} / \mathrm{s}^{2}$ \\
Plastic stiffness & $k_{1} / k$ & 0.2 & \\
Adhesion "stiffness" & $k_{c} / k$ & 1.0 & \\
Friction stiffness & $k_{t} / k$ & 0.2 & \\
Rolling stiffness & $k_{r} / k$ & 0.2 & \\
Torsion stiffness & $k_{o} / k$ & 0.2 & \\
\hline Plasticity depth & $\phi_{f}$ & 0.05 & \\
\hline Coulomb friction coefficient & $\mu=\mu_{d}=\mu_{s}$ & 1 & \\
Dynamic to static Friction ratio & $\phi_{d}=\mu_{d} / \mu_{s}$ & 1 & \\
Rolling "friction" coefficient & $\mu_{r}$ & 0.1 & \\
Torsion "friction" coefficient & $\mu_{o}$ & 0.1 & \\
\hline Normal viscosity & $\gamma=\gamma_{n}$ & $210^{-13} \mathrm{~kg} / \mathrm{s}$ & $210^{-7} \mathrm{~kg} / \mathrm{s}$ \\
Friction viscosity & $\gamma_{t} / \gamma$ & 0.25 & \\
Rolling viscosity & $\gamma_{r} / \gamma$ & 0.25 & \\
Torsion viscosity & $\gamma_{o} / \gamma$ & 0.25 & \\
Background viscosity & $\gamma_{b} / \gamma$ & 0.10 & \\
Background viscous torque & $\gamma_{b r} / \gamma$ & 0.05 & \\
\hline Lennard Jones energy & $\varepsilon$ & $0.10^{-15} \mathrm{~J}$ & $0.10^{-3} \mathrm{~J}$ \\
Lennard Jones distance & $r_{0} /(2 a)$ & 0.5 & \\
Lennard Jones cut-off & $r_{c} /(2 a)$ & 1.5 & \\
\hline
\end{tabular}

Table 1: The microscopic material parameter values used if not explicitly specified. The third column contains those values that are different due to rescaling of the unit of time, i.e., when seconds are read as $\mu s$.

A typical set of material parameters is given in table 1 . The choice of numbers and units is such that the particles correspond to micro-meter sized, (overly) soft aluminum spheres. The stiffness magnitude (this is not the material bulk modulus, but a contact property) used thus appears much too small for this material - however, dependent on the volume fraction (or the external) pressure, the material deformation (overlap) can be realistic if the simulations are performed so slow that rate effects are small and overlaps are not becoming too large. A simple rescaling of time brings the material parameters into the reasonable range - see rightmost column in table 1.

Using the parameter $k=k_{2}$ in Eq. (4) leads to a typical contact duration (halfperiod) $t_{c} \approx 2.2710^{-4} \mathrm{~s}$, for a normal collision with $\gamma=0$. Accordingly, an integration time-step of $t_{\mathrm{MD}}=210^{-6} \mathrm{~s}$ is used, in order to allow for a 'safe' integration of contacts. Note that not only the normal "eigenfrequency" but also the eigenfrequencies in tangential and rotation direction have to be considered as well as the viscous response times $t_{\gamma} \approx m / \gamma$. All of the eigenfrequencies should be considerably larger than $t_{\mathrm{MD}}$, whereas the viscous response times should be even larger, so that $t_{\gamma}>t_{c}>t_{\mathrm{MD}}$. The discussion of all the effects due to the interplay between the model parameters is far from the scope of this paper, however. 


\subsection{Compression simulations}

When compressing the system (by growing the particles) the first quantity of interest is the density (volume fraction) $\nu$. For a set of frictionless hard spheres, the maximum volume fraction is $\nu_{\max } \approx 0.74$, when all spheres are optimally arranged on a crystal lattice. Random packings can reach volume fractions between 0.63 and 0.69 , dependent on the degree of local crystallization. When friction is switched on and also the other force laws are used, much smaller volume fractions between 0.55 and 0.58 are expected as indicated by the difference between open and solid symbols in Fig. 2 (Left).

Before the results of the compression simulations are discussed, one has first to decide on a criterion whether a packing is stable and quasi-static or not. In the following, the ratio of kinetic to potential energy is used, $e=E_{k i n} / E_{p o t}$, and the densities are reported when $e=1,10^{-1}, 10^{-2}$, and $10^{-3}$, as given in the inset of Fig. 2. Since the particles are continuously growing, the system has no chance to become static at the lowest density (relaxation times are extremly large at this point). However, the decay of $e$ close to this point is very rapid and indicates at least the possibility of a static, stable packing configuration. A more detailed study of alternative boundary conditions is in progress ${ }^{2}$.

For fixed friction coefficient, $\mu=1$, increasing rolling- and torsion-coefficients lead to lower densities. For the higher values of $\mu_{r}$ and $\mu_{o}$, reorganization can appear more violently during ongoing compaction, leaving the system with somewhat higher density. For fixed finite rolling- and torsion coefficients, $\mu_{r}=\mu_{o}=0.1$, the density is close to the reference without tangential forces and torques. With increasing friction coefficient $\mu$ the density drops. But the highest values of $\mu \geq 0.5$ do not necessarily lead to lower densities, as one could have expected. Again, the more violent reorganization events could be responsible.
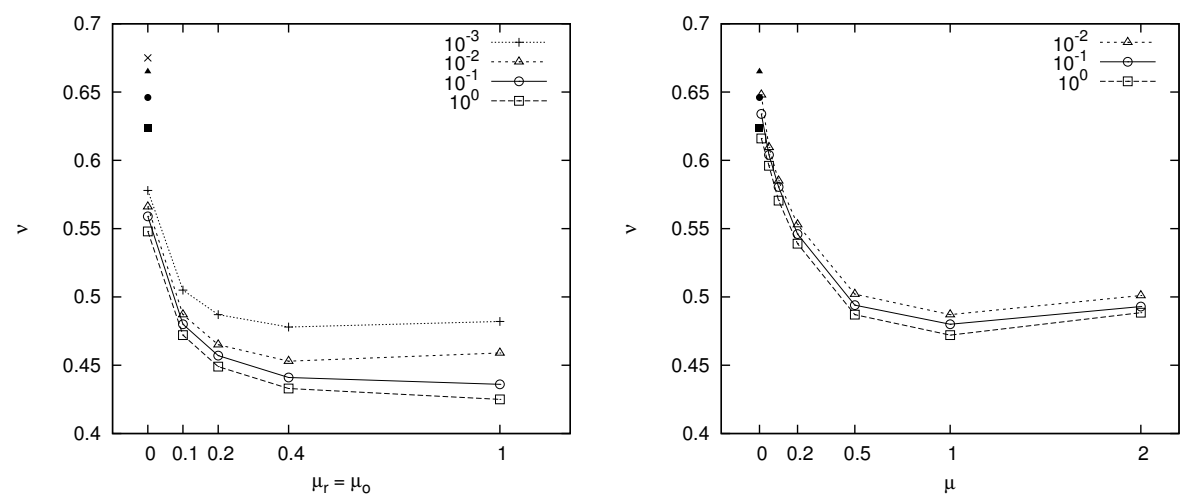

Figure 2: (Left) Densities (volume fractions) at which the energy ratio reaches the values $e$ as given in the inset. The parameters are given in table 1, only the values of rolling- and torsion-coefficients are varied while $m u=1$ is kept constant. The lines are a guide to the eye and the solid points are the reference data for $\mu=\mu_{r}=\mu_{o}=0$. (Right) Rolling and torsion-coefficients are $\mu_{r}=\mu_{o}=0.1$ and the friction coefficient $\mu$ is varied. Lines and solid points are the same as in the left panel.

\footnotetext{
${ }^{2}$ The simple approach of a system that is compressed by pressure controlled walls does not work, since the packing becomes then inhomogeneous: very low density in the center, higher density at the walls and very high densities in the corners.
} 
From various simulations with different material parameters (data not shown), one can conclude that higher viscosity parameters lead to faster relaxation - too high viscosity on the other hand leads to spurious effects. As long as the growth rate was small enough, the achieved low densities were comparable in magnitude, with no evident dependency on the viscosity parameters. Note, however, that all viscosities were active in normal, tangential, rolling, and torsion direction as well as for the background damping. The relative magnitude of the viscosities, as given in table 1, is found reasonable; however, a more systematic study might reveal a better, more realistic, combination of viscosity values.

Based on the variation of the friction-, rolling-, and torsion-coefficients, the lowest volume fraction to be expected for a stable packing can be extrapolated from Fig. 2 (Left) to be about $\nu_{\min } \approx 0.42$. Too small friction coefficients are always related to rather high densities. On the other hand, extremely high friction-coefficients do not necessarily lead to lower densities due to a different reorganization dynamics. A test simulation with $\mu=\mu_{r}=\mu_{o}=10$ did not lead to lower densities as one could have hoped.

\subsection{Compaction with long range force}

In order to achieve even lower densities as in the previous subsection, all parameters from table 1 are used, only the Lennard-Jones energy parameter is varied using the values $\varepsilon=10^{-21}, 10^{-20}, 10^{-19}, 10^{-18} \mathrm{~m}^{2} \mathrm{~kg} \mathrm{~s}^{-2}$. The potential energy now also involves the longrange potential and is thus not an adequate criterion for comparison to the kinetic energy. From the kinetic energy, the simulation with $\varepsilon=10^{-20} \mathrm{~m}^{2} \mathrm{~kg} \mathrm{~s}^{-2}$ seemed to relax most rapidly. The minimal density found was $\nu_{\min } \approx 0.34-$ further detailed studies about the value where the packing remains stable are necessary.

A qualitative comparison of the packing structures in Fig. 3 shows somewhat larger holes in the packing with long range forces (visible due to the dark particles in the back). A more quantitative analysis using the pair-correlation function reveals that a considerable fraction of particles that are close to each other without longrange forces stick together when long range forces are active. More detailed parameter studies are in progress.

Thus with some attractive long range force, the minimal packing density can be considerably decreased relative to the lowest densities achieved with contact forces only. However, very strong attractive forces do not necessarily lead to better relaxation behavior. Therefore, more detailed studies under better controlled conditions will be necessary to achieve stable configurations with extremely low density and to better understand the compaction and reorganization dynamics.

\section{Conclusion}

The present study is a summary of the soft particle force models involving elasticity, plastic contact deformation, adhesion, friction, and rolling- as well as torsion resistance. A set of parameters is given and several criteria and rules for parameter selection are discussed. Using friction and rolling-/torsion-resistance, stable static packings could be reached with densities (volume fractions) somewhat above $\nu_{\min } \approx 0.4$. When also an attractive, longer ranging force was added, the minimal possible density was below $\nu_{\min } \approx 0.34$, however, in this case the criterion for a static, stable configuration has to be improved. 

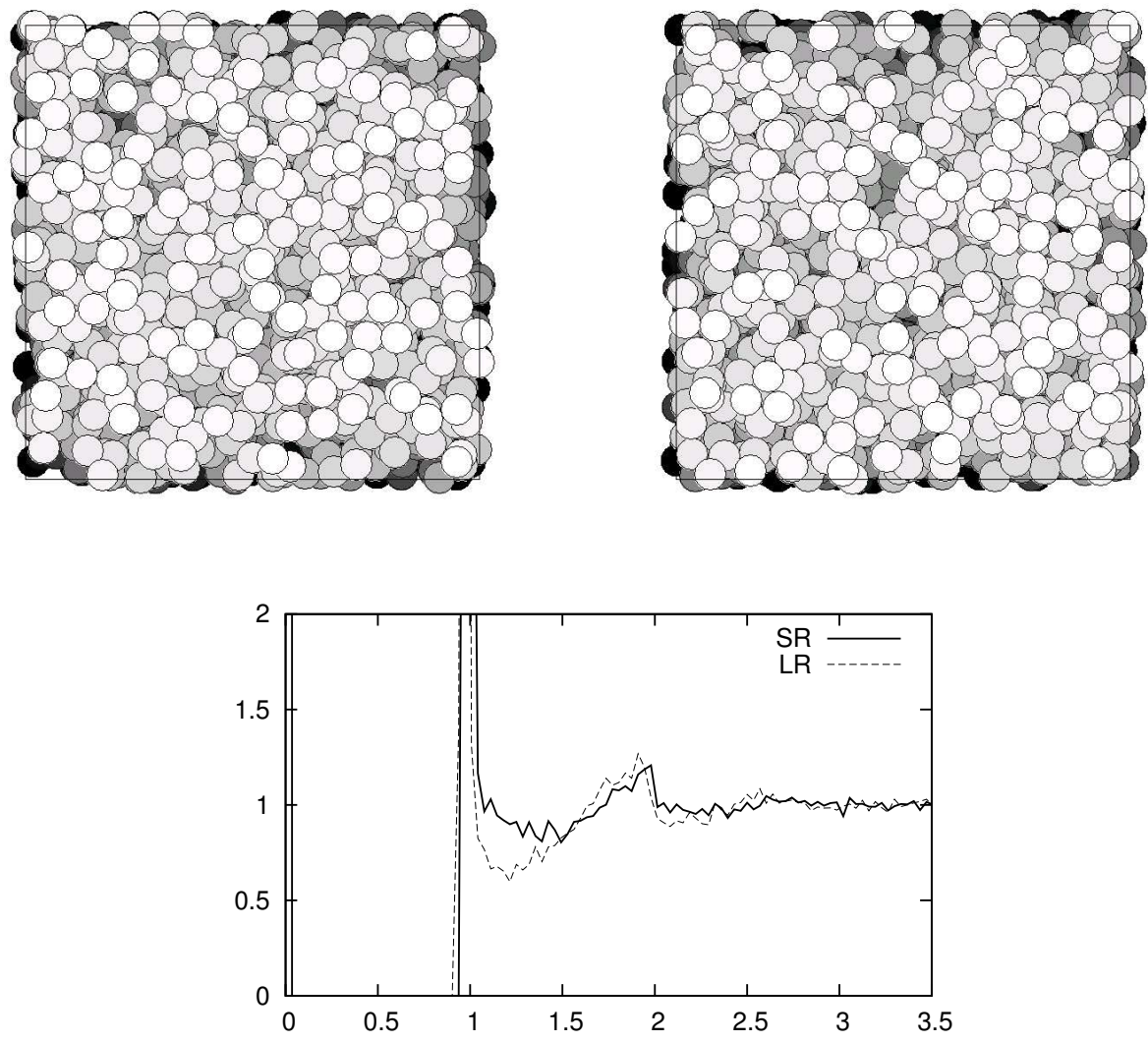

Figure 3: Snapshots from simulations without (Left) and with (Right) longer ranged attractive forces at volume fraction $\nu=0.34$. The left packing is not yet stable at this density whereas the right packing is. The greyscale indicates the distance from the viewer - more distant particles are darker. The lines indicate the (periodic) boundary of the system; note that dark particles at the system boundaries are visible due to the periodic boundary conditions - not due to holes in the system. (Bottom) Pair correlation functions for the simulations with (LR) and without (SR) longrange forces.

Even though molecular dynamics particle methods are a helpful tool for the understanding of granular systems, the quality of the results depends strongly on the contact models used. The set of contact models presented here, besides many model assumptions, still involves a large number of parameters. Some of them are less important for physical properties and behavior of the system than others - the latter, most relevant parameters have to be identified and their interplay has to be better understood.

The qualitative particle-modeling approach of the early years has now developed into the attempt of a quantitative predictive modeling of the diverse modes of complex behavior in granular media. The quantitative comparison with experiments and validation of the models is the task for the near future. The measurement of low packing fractions in adhesive, frictional fine powders is one of the possible experiments to be examined in more detail - a challenge for particle contact modeling. 


\section{Acknowledgements}

Besides valuable discussions with many colleagues, we acknowledge the financial support of the Deutsche Forschungsgemeinschaft (DFG) and the Stichting voor Fundamenteel Onderzoek der Materie (FOM), financially supported by the Nederlandse Organisatie voor Wetenschappelijk Onderzoek (NWO).

\section{References}

[1] P. A. Vermeer, S. Diebels, W. Ehlers, H. J. Herrmann, S. Luding, and E. Ramm, editors. Continuous and Discontinuous Modelling of Cohesive Frictional Materials, Berlin, 2001. Springer. Lecture Notes in Physics 568.

[2] P. A. Cundall and O. D. L. Strack. A discrete numerical model for granular assemblies. Géotechnique, 29(1):47-65, 1979.

[3] Y. M. Bashir and J. D. Goddard. A novel simulation method for the quasi-static mechanics of granular assemblages. J. Rheol., 35(5):849-885, 1991.

[4] H. J. Herrmann, J.-P. Hovi, and S. Luding, editors. Physics of dry granular media - NATO ASI Series E 350, Dordrecht, 1998. Kluwer Academic Publishers.

[5] C. Thornton. Numerical simulations of deviatoric shear deformation of granular media. Géotechnique, 50(1):43-53, 2000.

[6] C. Thornton and L. Zhang. A dem comparison of different shear testing devices. In Y. Kishino, editor, Powders \& Grains 2001, pages 183-190, Rotterdam, 2001. Balkema.

[7] M. Lätzel, S. Luding, H. J. Herrmann, D. W. Howell, and R. P. Behringer. Comparing simulation and experiment of a $2 \mathrm{~d}$ granular couette shear device. Eur. Phys. J. E, 11(4):325-333, 2003.

[8] M. P. Allen and D. J. Tildesley. Computer Simulation of Liquids. Oxford University Press, Oxford, 1987.

[9] D. C. Rapaport. The Art of Molecular Dynamics Simulation. Cambridge University Press, Cambridge, 1995.

[10] S. Luding. Collisions \& contacts between two particles. In H. J. Herrmann, J.-P. Hovi, and S. Luding, editors, Physics of dry granular media - NATO ASI Series E350, page 285, Dordrecht, 1998. Kluwer Academic Publishers.

[11] O. R. Walton and R. L. Braun. Viscosity, granular-temperature, and stress calculations for shearing assemblies of inelastic, frictional disks. J. Rheol., 30(5):949980, 1986.

[12] Jürgen Tomas. Particle adhesion fundamentals and bulk powder consolidation. KONA, 18:157-169, 2000.

[13] C. Y. Zhu, A. Shukla, and M. H. Sadd. Prediction of dynamic contact loads in granular assemblies. J. of Applied Mechanics, 58:341, 1991. 
[14] M. H. Sadd, Q. M. Tai, and A. Shukla. Contact law effects on wave propagation in particulate materials using distinct element modeling. Int. J. Non-Linear Mechanics, 28(2):251, 1993.

[15] S. Luding, K. Manetsberger, and J. Müllers. A disrete model for long time sintering. Journal of the Mechanics and Physics of Solids, 53(2):455, 2005.

[16] S. Luding, E. Clément, A. Blumen, J. Rajchenbach, and J. Duran. The onset of convection in molecular dynamics simulations of grains. Phys. Rev. E, 50:R1762, 1994.

[17] G. Bartels, T. Unger, D. Kadau, D. E. Wolf, and J. Kertesz. The effect of contact torques on porosity of cohesive powders. Granular Matter, 7:139, 2005.

[18] E. Dintwa, M. van Zeebroeck, E. Tijskens, and H. Ramon. Torsion of viscoelastic spheres in contact. Granular Matter, 7:169, 2005.

[19] S. Luding, E. Clément, A. Blumen, J. Rajchenbach, and J. Duran. Anomalous energy dissipation in molecular dynamics simulations of grains: The "detachment effect”. Phys. Rev. E, 50:4113, 1994. 